\title{
A Novel Analog Modulation Classification: Discrete Wavelet Transform- Extreme Learning Machine (DWT-ELM)
}

\author{
Mehmet USTUNDAG* \\ Malatya Turgut Ozal University, Faculty of Engineering and Natural Sciences, \\ Department of Electrical-Electronics Engineering, Turkey \\ (ORCID: 0000-0003-4936-7690)
}

\begin{abstract}
The aim of this study is to propose a method using discrete wavelet transform and extreme learning machine (DWT-ELM) in classification of communication signals. Six types of analog modulated signals as "AM", "DSB", "USB", "LSB", "FM" and "PM" are used for classification and analog modulated signal dataset consists of 1920 signals. These signals are also added white noise. Feature extraction is performed using different DWT filters. The feature vector obtained from DWT is used in classification. ELM is preferred due to its advantages over conventional back-propagation based classification. The feature vector is fed by the inputs of the ELM. The performance of the proposed method is evaluated for different types of DWT filters. In addition, compared results with similar study are presented to be able to determine the success of the proposed method.
\end{abstract}

Keywords: DWT-ELM, ELM classification, Wavelet Transform, Analog modulated signals.

\section{Yeni Bir Analog Modülasyon Sınıflandırması: Ayrık Dalgacık Dönüşümü- Aşırı Öğrenme Makinesi (ADD-AÖM)}

\begin{abstract}
$\ddot{O} \mathbf{z}$
$\mathrm{Bu}$ çalışma, analog modüle edilmiş iletişim sinyallerinin sınıflandırılması için ayrık dalgacık dönüşümü - aşırı öğrenme makinesine (ADD-AÖM) dayalı yeni bir yöntem önermektedir. Sınıflandırma için AM, DSB, USB, LSB, FM ve PM olmak üzere altı tip analog modüle edilmiş sinyal kullanılır ve analog modüle edilmişs sinyal veri seti 1920 sinyalden oluşur. Bu sinyallere ayrıca beyaz gürültü eklenir. Özellik çıarma işlemi, farklı ADD filtreleri kullanılarak gerçekleştirilir. ADD'den elde edilen öznitelik vektörü sınıflandırmada kullanılır. AÖM, geleneksel geri yayılmaya dayalı sınıflandırmaya göre avantajları nedeniyle tercih edilmektedir. Özellik vektörü, AÖM sınıflandırıcısının girişine beslenir. Önerilen yöntemin performansı, farklı ADD filtreleri için değerlendirilir. Ayrıca, önerilen yöntemin performansını değerlendirmek için benzer çalışma ile karşılaştırılan sonuçlar sunulmuştur.
\end{abstract}

Anahtar kelimeler: ADD-AÖM, AÖM sınıflandırma, Dalgacık Dönüşümü, Modüle edilmiş analog sinyaller.

\section{Introduction}

The analog modulated communication signal (AMCS) classification is stage between detection and demodulation of a signal. It is widely used in various fields, such as civil and military applications. In earlier studies on analog modulated communication signal classification, the measured parameters had been interpreted by an expert. The classification of AMCS has been made in three ways: classical methods, semi-automatic methods and automatic methods. In the classic method, spectrum feature, instantaneous amplitude and phase of the signal to be analysed are evaluated by human. The designed demodulator for one of each modulation type was utilized in the semi-automatic methods. Automatic methods for analog modulated communication signal classification automatically decide the modulation

"Corresponding author: mehmet.ustundag@ozal.edu.tr

Received: 03.01.2021, Accepted: 19.03.2021 
type. Automatic analog modulation classification methods can be divided: "decision-theoretic", "statistical pattern recognition" approaches and intelligent system based approaches.

There have been existed some studies in literature on automatic classification of the AMCS. Ref. [1] proposes a modulation classifier using instantaneous frequency and amplitude. The proposed method can distinguish between "AM", "FM" and "DSB" signals. Ref. [2] presented a modulation method based envelope characteristic of the signal for classification of "AM", "FM", "DSB" and "SSB" analog modulation types. Ref. [3] a method has been proposed to classify analog radio signals. A method for analog modulation classification is presented to distinguish AM and noisy environment in Ref. [4]. A modulation classifier is presented to distinguish "USB" and "LSB" in Ref. [5]. To recognize the modulation type, the presented method uses instantaneous frequencies of these signals. In Ref. [6], a modulation classifier is proposed for recognition of the modulation kinds such as "AM", "DSB", "VSB", "LSB", "USB", "FM" and mixed modulation signals. Ref. [7] suggests a method based on decision theoretic approach. Ref. [8-10] present an automatic method based on ANN for modulation classifier. Ref. [11] proposes a new modulation classification method based on discrete wavelet neural network.

This study proposes a novel automatic analog modulation method based on DWT-ELM for recognition of the six modulation types. The modulation types are "AM", "DSB", "USB", "LSB", "FM" and "PM". 1920 AMCS are generated by MATLAB Communication Toolbox and they are used for the training and testing of the proposed method. Performance of the proposed method is evaluated for different wavelet filter families such as "Symlets", "Daubechies", "Biorthogonals", and "Coiflets". Further, the results of the proposed method are compared with Ref. [11]. The obtained classification results show that the proposed method has better classification performance over previous studies.

This paper: Section 2 explains analog modulated signals, section 3 and section 4 presents DWT and ELM, respectively. The proposed method is given in Section 5. Application of the proposed method and obtained results is presented in Section 6. Finally, shows the results in Section 7.

\section{Material and Methods}

\subsection{Analog Modulated Signals (AMS)}

An AMS $f(t)$ can be formulated as follows [11]:

$$
f(t)=e_{c} s(t) \cos \left(2 \pi f_{c} t+\varphi(t)+\theta_{0}\right)
$$

Here; $f_{c}$ is carrier frequency, $\varphi(t)$ is phase of signal, $\mathrm{s}(t)$ is signal envelope and $\theta_{0}$ is initial phase of signal. It is shown that $e_{c}$ controls power of the carrier signal. Six AMS types used in this study is presented as follows [11]:

(Transmission carrier amplitude modulation "AM"); The equation for this signal is shown below:

$$
f(t)=1+m \cdot f(t) \cdot \cos \left(2 \pi f_{c} t\right)
$$

(Double sideband with supress carrier AM "DSB"); The equation for this signal is shown below:

$f(t)=f(t) \cdot \cos \left(2 \pi f_{c} t\right)$

(Upper side band modulation "USB"); The equation for this signal is shown below:

$$
f(t)=f(t) \cdot \cos \left(2 \pi f_{c} t\right)-h(t) \cdot \sin \left(2 \pi f_{c} t\right)
$$

(Lower side band modulation "LSB"); The equation for this signal is shown below:

$$
f(t)=f(t) \cdot \cos \left(2 \pi f_{c} t\right)+h(t) \cdot \sin \left(2 \pi f_{c} t\right)
$$

(Frequency modulation "FM"); The equation for this signal is shown below: 


$$
f(t)=\cos \left(2 \pi f_{c} t+K_{f} \int_{-\infty}^{t} x(\tau) d \tau\right)
$$

(Phase modulation "PM"); The equation for this signal is shown below:

$$
f(t)=\cos \left(2 \pi f_{c} t+2 \pi \varphi(t)+\theta_{0}\right)
$$

\subsection{Discrete Wavelet Transform (DWT)}

Basic feature extraction constitutes an important step in pattern classification. Time and frequency information of AMS is important. Since the modulated signal may contain different components, it is correct to separate the signal into "detail components" [12]. The DWT analyzes the signal with various resolutions by decomposing the approximate and detailed coefficient signals in various frequency bands, so it is often used in the model classification [12].

The DWT consists of two parts as shown in Figure 1. These are low and high pass filters. After the modulated signal is applied to these filters, approximate and detail coefficients are obtained.

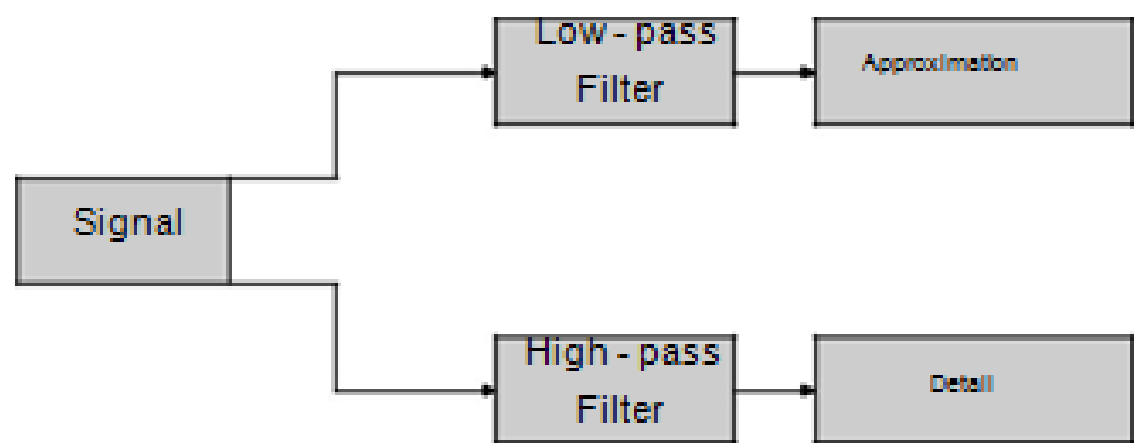

Figure 1. DWT decomposition structure

In the DWT method, when resolving high frequency components in a minor window, they need large time windows to solve low frequency components. Because the signal has low and high frequency components. At the same time, components with high frequency are represented in smaller time intervals, while components with low frequency are represented in larger time intervals. The equality of DWT function is shown below [13]:

$$
d_{m}\left(t_{m}\right)=x(t) \psi_{m}\left(\frac{t-t_{m}}{2^{m}}\right)
$$

In this equation, " $\psi_{m}$ " is the $m$ level decomposition filter. In DWT transformation, it is divided into its components as $2^{m}$ according to the value of $m$. Time-frequency coefficients obtained from the signal [14]:

$$
d[n]=x[n] h[n], \quad c[n]=x[n] g[n]
$$

In Equation 9, “ $h[n]$ "is the "impulse respons"e of the "high-pass filter", " $g[n]$ "is the "impulse response" of the "low-pass filter" $[12,15,16]$.

\subsection{Extreme Learning Machine}

ELM can be called a single hidden layer feed forward network without a neuron in its hidden layer [17], [18]. For the ELM method; Output function for "Single Hidden Layer Feed Forward Neural Networks" (SLFN) is given below. 


$$
f_{L}(x)=\sum_{i=1}^{L} \beta_{i} h_{i}(x)=h(x) \beta
$$

In the equation; $\beta=\left[\beta_{1}, \ldots, \beta_{L}\right]^{T}$ is the output weight vector. $h(x)=(h l(x) \ldots, h L(x)]$ is the feature mapping of the equation ELM. The hidden neurons used in ELM may have different output functions and they may not be unique.

ELM uses two steps when training the SLFN. These are linear parameter decoding and random feature mapping. The ELM uses some nonlinear mapping functions to initialize the hidden layer, thus extracting the feature space for input data. The random feature differs from current learning algorithms, such as deep neural networks [19], that use the mapping phase, SVM, Auto (Encoders/Decoders). The mapping functions used in the ELM method can be nonlinear piecewise continuous functions.

ELM is more efficient than conventional Back-Propagation (BP) neural networks. The reason for this is that hidden node parameters used in ELM are randomly generated. Randomly generated nodes were created according to the probability distribution.

Other than the filter functions listed in Table 2, there are also functions such as fuzzy ELM [2022] and wavelet ELM [23-26]. In the next step, the weights denoted by and combining the hidden layer and the output layer are solved by reducing the approximate error to the square error.

$$
\min _{\beta \in \mathbf{R}^{L \times m}}\|\mathbf{H} \beta-\mathbf{T}\|^{2}
$$

Here "H" is the "hidden layer" output matrix given in the Eq. (12):

$$
\mathbf{H}=\left[\begin{array}{c}
h\left(x_{1}\right) \\
\vdots \\
h\left(x_{N}\right)
\end{array}\right]=\left[\begin{array}{ccc}
h_{1}\left(x_{1}\right) & \ldots & h_{L}\left(x_{1}\right) \\
\vdots & \vdots & \vdots \\
h_{1}\left(x_{N}\right) & \vdots & h_{L}\left(x_{N}\right)
\end{array}\right]
$$

This symbol $(\|\|$.$) is used to show the Frobenius norm. The most appropriate solution for Eq.$ (11) is given below:

$$
\beta^{*}=\mathrm{H}^{\dagger} \mathrm{T}
$$

The " $\mathrm{H}$ " given here indicates the generalized inverse of the " $\mathrm{H}$ " matrix with Moore-Penrose. There are many effective methods to solve the problem given in the above equation, such as "orthogonal projection method", Gaussian elimination, "iterative method" and "single value decomposition (SVD)" etc. [27].

The difference of "ELM" from traditional learning algorithms is to simultaneously satisfy a few salient targets [28-30]. Generalization performance is not considered in most algorithms when the feedforward neural networks are proposed for the first time.

\subsection{Proposed Method}

In this paper, AMS dataset is created by MATLAB Communication Toolbox. In these signals, "a message signal", which is a real "voice signal" with band-limited to $4 \mathrm{kHz}$, is used. Extensive simulation studies are performed to produce analog-modulated signals using different values of signal parameters such as phase, modulation index, "Signal-to-Noise Ratio" (SNR), and frequency deviation in FM, phase deviation in PM. The 320 signals are obtained for each of analog-modulation signals by means of simulation studies. The details of analog modulated signal generation are given in Table 1 .

The steps of the proposed method is given in Figure 2. The proposed method can be divided into four steps: signal generation, feature extraction, training and testing. 


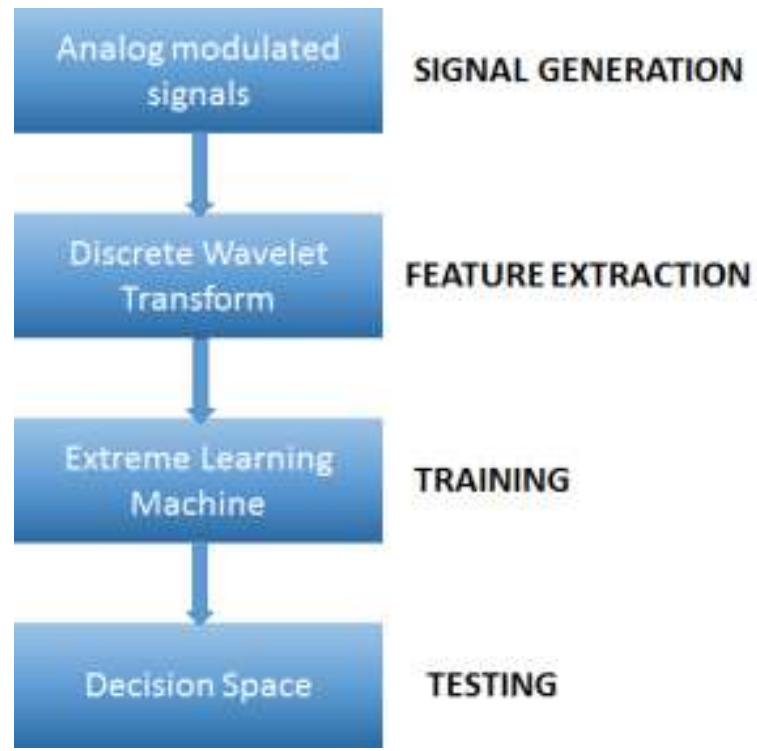

Figure 2. Steps of the proposed method

1920 AMS are created by using functions in "MATLAB Communication Toolbox" for "AM", "DSB", "USB", "LSB", "FM", and "PM". In the feature extraction step, the DWT is applied to the AMS. Tree structure with 7-level was used in DWT decomposition. Daubechies wavelets ("db2", "db3", "db5", "db8", "db10"), "Symlets wavelets" ("sym2", "sym3", "sym5", "sym7”, "sym8"), "Biorthogonal wavelets" ("bior1.3", "bior2.2", "bior2.8", "bior3.5", "bior6.8") and "Coiflets wavelets" ("coif1", "coif2", "coif3", "coif4", and "coif5") are used in DWT decomposition. Thus, a comparison study is performed by using different wavelet filter type. After the AMS are separated into their components by filters, seven detail coefficients and one approximation coefficient are obtained. Then, the adaptive wavelet norm entropy $E(s)$ given in Eq.14 is computed for each of "DWT coefficients".

$$
E(s)=\frac{\sum_{i}\left|s_{i}\right|^{p}}{N}
$$

Here, $s$ is terminal node signal, and $p$ is power. In this study, $p$ parameter value is selected between (12) and its value is update by step increments of 0.1 . Further, $p$ parameter is normalized by using its maximum value. The real voice signal used in this study are depicted in Figure 3. "DWT" of an "AM" modulated signal are given in Figure 3 for "db2", "sym5", "bior6.8", and "coif3" wavelet filters.

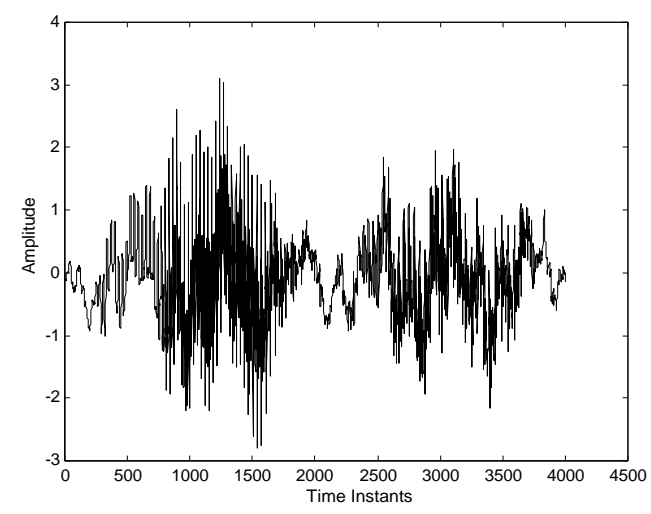

Figure 3. Real voice signal 


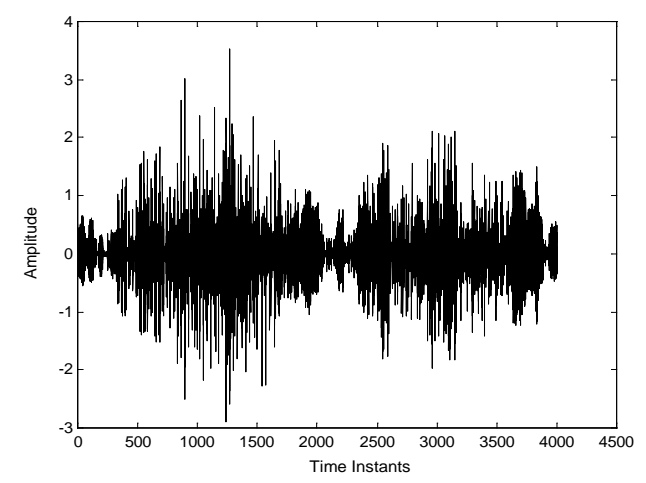

Figure 4. AM modulated signal with " $m=0.5$ ", and " $\theta_{0}=0$ " in Table 2.

The feature extraction method (FEM) for different wavelet filter types used in this study is given

In this study, ELM is preferred as classifier due to its superiority over traditional backpropagation algorithms. Tangent sigmoid is used in the "activation function" preference.

\section{Results}

The experiments are performed for classification of the six analog modulated signals. For this aim, 320 signals are generated for each analog modulated signals. 50\% of analog modulated signals (960 signals) is used for training of the ELM classifier and remaining signals (960 signals) for testing of the ELM. These training and testing of the classifier are repeated for each of FEMs. In Table 3, the obtained results are given in terms of "training time" and "classification accuracy" for each FEM. This table shows that FEM-18 reaches to "desired error value" in the "shortest time" whereas FEM-17 reaches longest time. FEM-2 has the best training performance among other FEMs and FEM-18 has the worst training performance. $p$ parameter value is 1 at the end of update for FEM-2. Updated $p$ parameter value is 1.1 for FEM-18. In Table 3, HNN is hidden neuron number of ELM, TEN is training epoch number, the LVP is last value of $p$ parameter, MSE is mean square error and TA is percent testing accuracy.
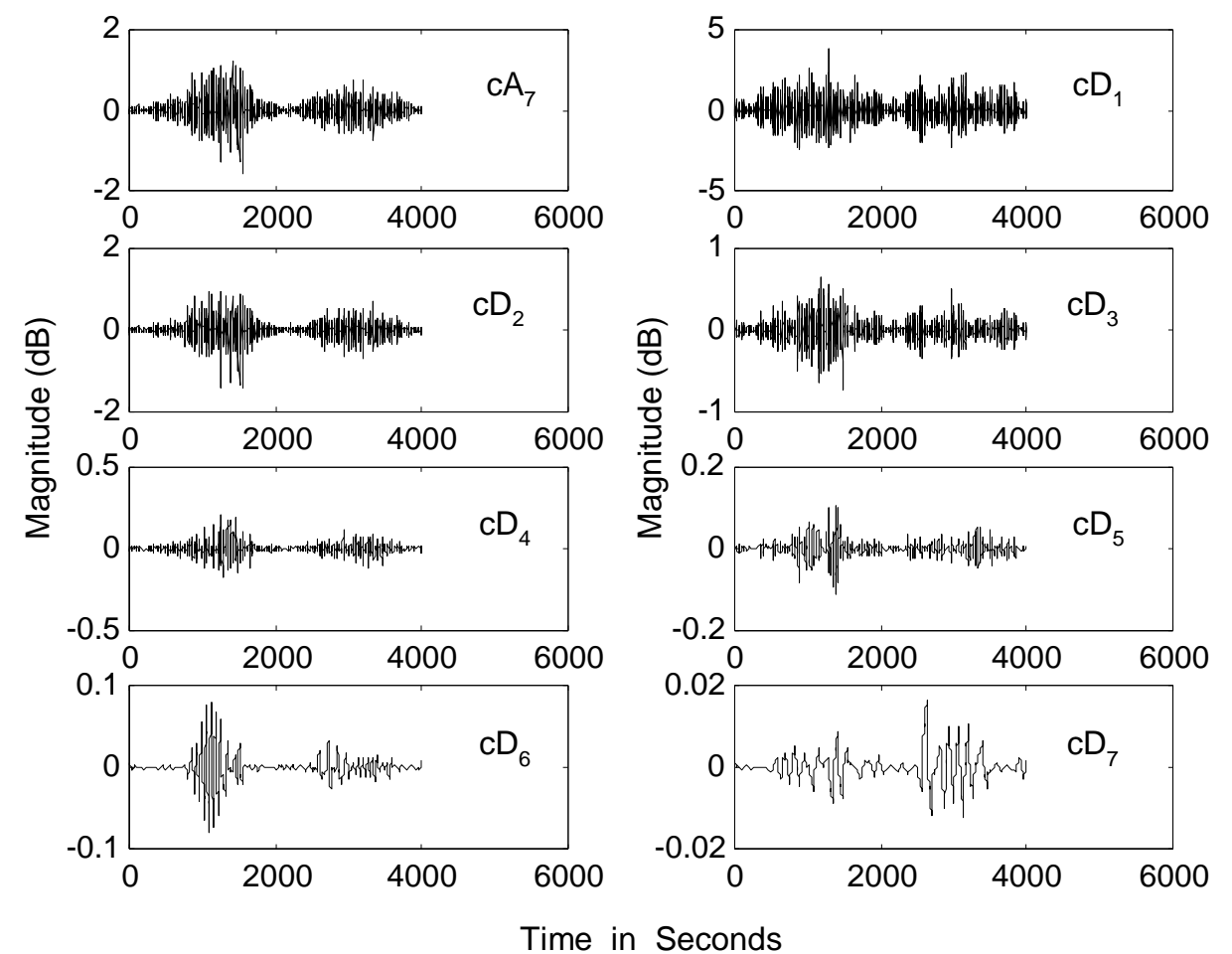

Figure 5. Waveforms showing the detail and approximate coefficients of the AM signal up to the 7th level of decomposition (“db2” wavelet filters) 

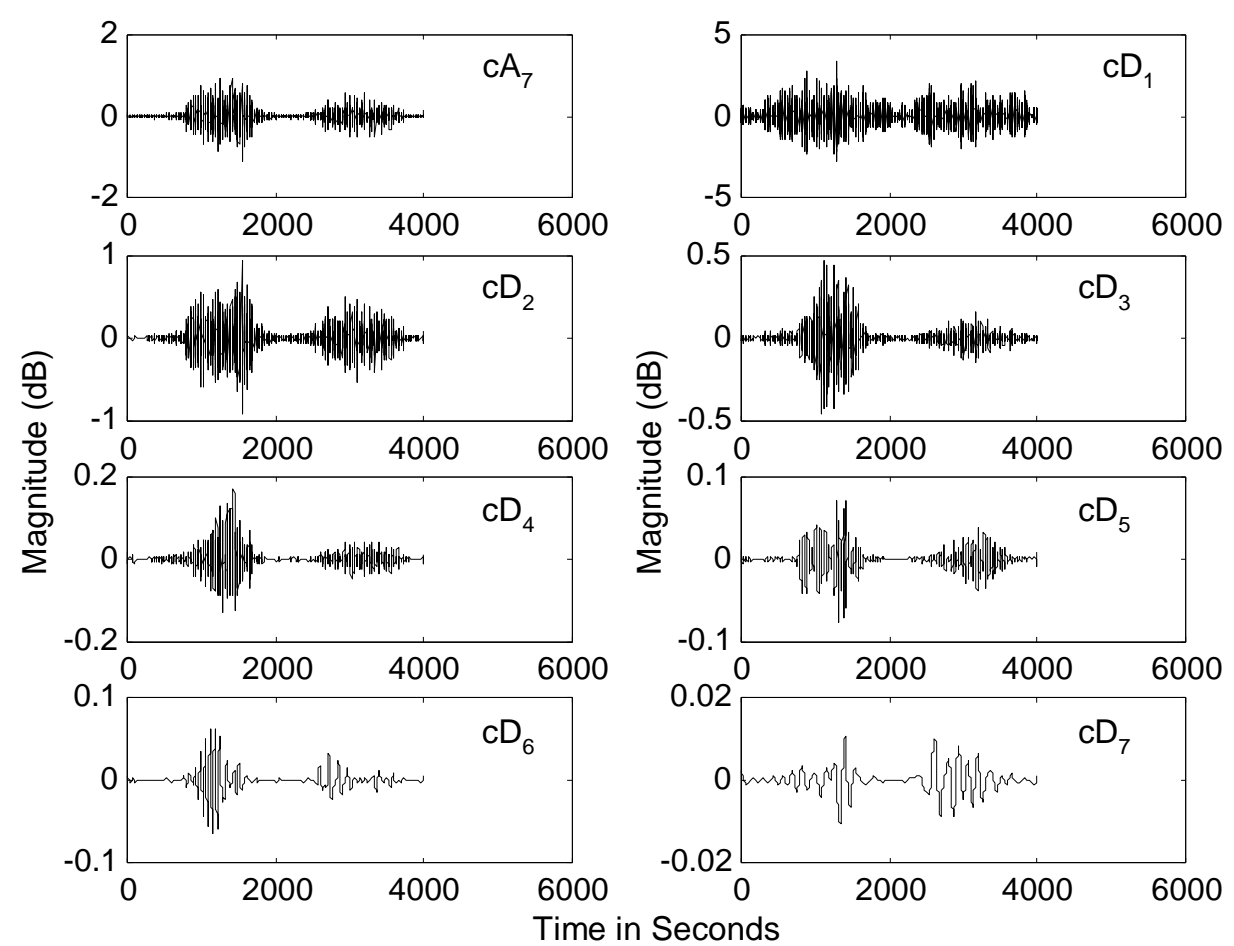

Figure 6. Waveforms showing the detail and approximate coefficients of the AM signal up to the 7th level of decomposition ("sym5" wavelet filters)
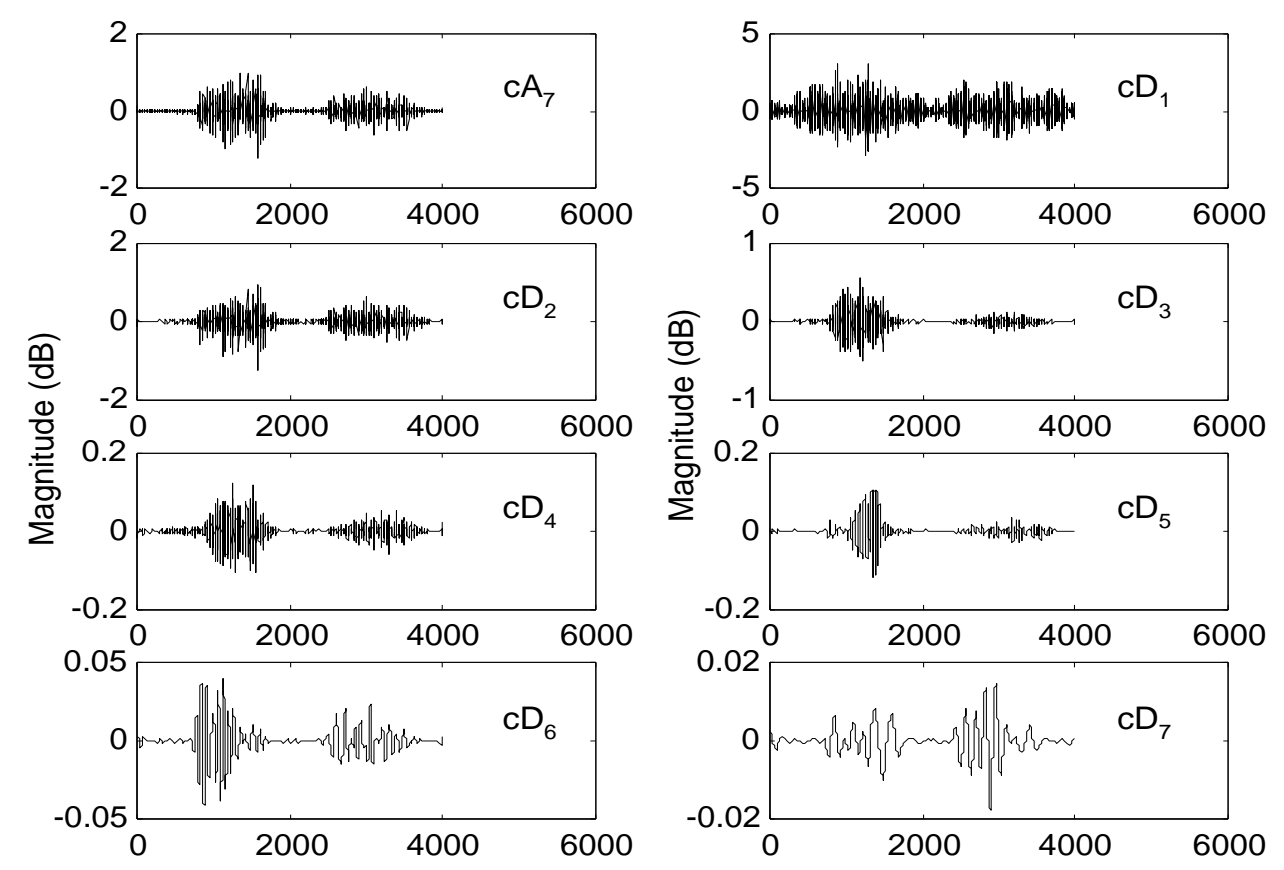

Time in Seconds

Figure 7. Waveforms showing the detail and approximate coefficients of the AM signal up to the 7th level of decomposition ("bior6.8" wavelet filters) 


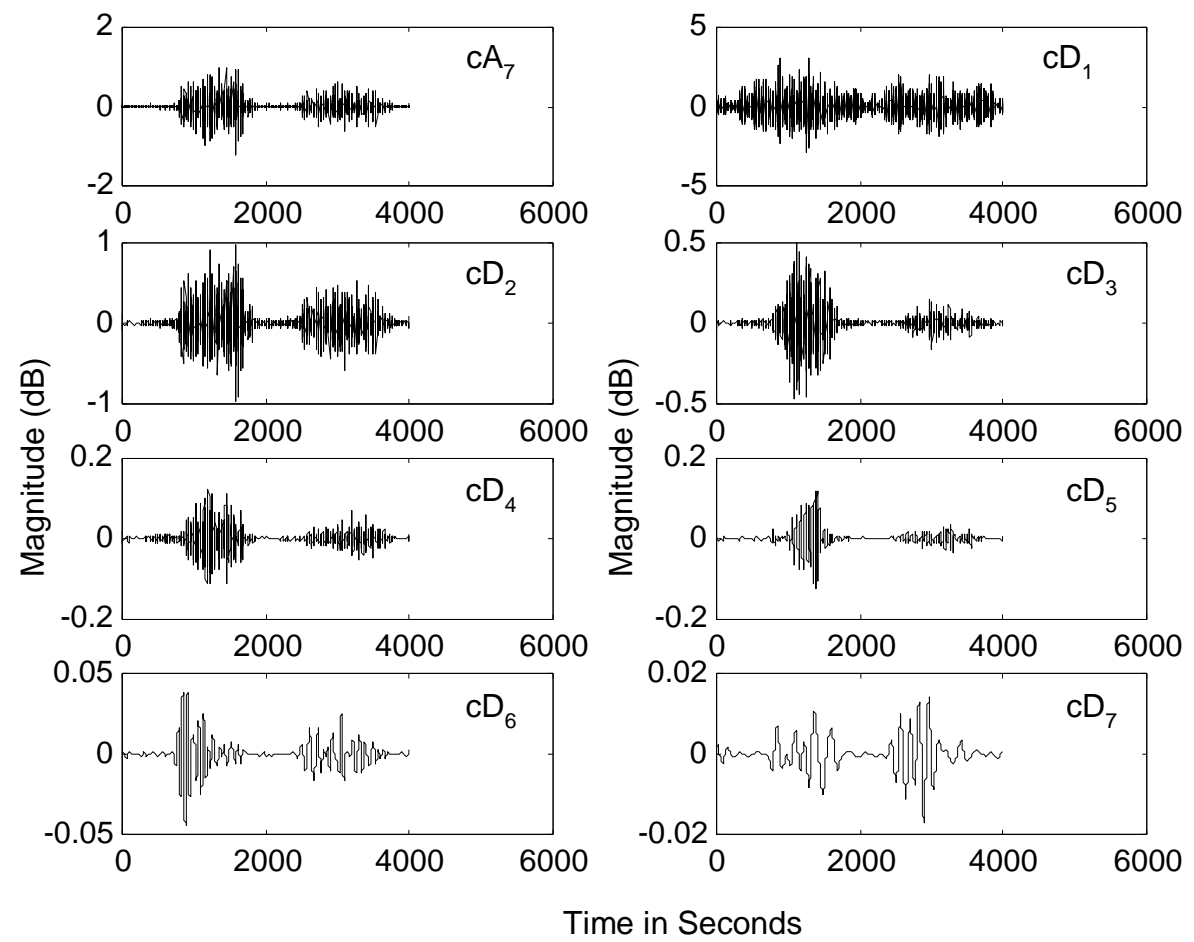

Figure 8. Waveforms showing the detail and approximate coefficients of the AM signal up to the 7th level of decomposition ("coif3" wavelet filters)

Table 1. The details of AMS generation

\begin{tabular}{cccccc}
\hline $\begin{array}{c}\text { Signal } \\
\text { type }\end{array}$ & Modulation index & $\begin{array}{c}\text { White Gaussian noise } \\
\text { with SNR }\end{array}$ & initial phase & Phase deviation & Total \\
\hline "AM" & $0.2,0.5$ and 1 & Between $0-60 \mathrm{~dB}$ & $0, \pi / 6, \pi / 2$ & - & 320 \\
"DSB" & - & Between $0-60 \mathrm{~dB}$ & $0, \pi / 6, \pi / 2$ & - & 320 \\
"USB" & - & Between 0 - $60 \mathrm{~dB}$ & $0, \pi / 6, \pi / 2$ & - & 320 \\
"LSB" & - & Between 0 - $60 \mathrm{~dB}$ & $0, \pi / 6, \pi / 2$ & - & 320 \\
"FM" & 1.5 and 10 & Between 0 - $60 \mathrm{~dB}$ & $0, \pi / 6, \pi / 2$ & - & 320 \\
"PM" & - & Between 0 - $60 \mathrm{~dB}$ & $0, \pi / 6, \pi / 2$ & $\pi / 9, \pi / 12, \pi / 18$ & 320 \\
\hline
\end{tabular}

Table 2. FEM for different wavelet filter types used in this study

\begin{tabular}{lccc}
\hline & Wavelet filter type & FEM & Wavelet filter type \\
\hline "FEM-1" & "db2" & "FEM-11" & "bior1.3" \\
"FEM-2" & "db3" & "FEM-12" & "bior2.2" \\
"FEM-3" & "db5" & "FEM-13" & "bior2.8" \\
"FEM-4" & "db8" & "FEM-14" & "bior3.5" \\
"FEM-5" & "db10" & "FEM-15" & "bior6.8" \\
"FEM-6" & "sym2" & "FEM-16" & "coif1" \\
"FEM-7" & "sym3" & "FEM-17" & "coif2" \\
"FEM-8" & "sym5" & "FEM-18" & "coif3" \\
"FEM-9" & "sym7" & "FEM-19" & "coif4" \\
"FEM-10" & "sym8" & "FEM-20" & "coif5" \\
\hline
\end{tabular}

Table 4 depict the "classification performance" of the FEM-2. As seen clearly from Table 4, two "AM signals" are classified as "LSB" and one "AM" signals as "DSB". 157 "AM" signals have been correctly classified. The classification performance of other FEMs are illustrated in Tables 5-23. 
Table 3. Training time and classification accuracy for different FEMs

\begin{tabular}{cccccc}
\hline FEM & HNN & TEN & LVP & MSE & TA \\
\hline FEM-1 & 60 & 781 & 1.2 & 0.00000190 & 96.45 \\
FEM-2 & 80 & 142 & 1 & 0.00000050 & 99.27 \\
FEM-3 & 80 & 672 & 1.1 & 0.00000450 & 96.66 \\
FEM-4 & 120 & 354 & 1.3 & 0.00000053 & 96.14 \\
FEM-5 & 40 & 1132 & 1.5 & 0.00000153 & 95.52 \\
FEM-6 & 60 & 784 & 1 & 0.00000451 & 95.52 \\
FEM-7 & 40 & 537 & 1 & 0.00000130 & 95.93 \\
FEM-8 & 30 & 2044 & 1.3 & 0.00000530 & 96.66 \\
FEM-9 & 60 & 632 & 1.2 & 0.00000826 & 97.29 \\
FEM-10 & 80 & 636 & 1.1 & 0.00000637 & 97.08 \\
FEM-11 & 50 & 872 & 1.3 & 0.00000536 & 95.93 \\
FEM-12 & 60 & 845 & 1.3 & 0.00001638 & 96.56 \\
FEM-13 & 80 & 726 & 1.2 & 0.00000647 & 95.41 \\
FEM-14 & 60 & 937 & 1.1 & 0.00003256 & 95.93 \\
FEM-15 & 30 & 546 & 1.1 & 0.00001738 & 95.52 \\
FEM-16 & 40 & 382 & 1.2 & 0.00000345 & 96.14 \\
FEM-17 & 100 & 3163 & 1.1 & 0.00000748 & 95.62 \\
FEM-18 & 80 & 76 & 1.1 & 0.00001839 & 95.20 \\
FEM-19 & 80 & 373 & 1.3 & 0.00002673 & 96.04 \\
FEM-20 & 60 & 537 & 1 & 0.00000378 & 96.56 \\
\hline
\end{tabular}

Table 4. Performance of the proposed method for FEM-2

\begin{tabular}{ccccccc}
\hline \multirow{2}{*}{ Actual } & \multicolumn{7}{c}{ Estimated } \\
\cline { 2 - 7 } & "AM" & "DSB" & "USB" & "LSB" & "FM" & "PM" \\
\hline "AM" & 159 & 1 & - & - & - & - \\
"DSB" & 1 & 158 & - & 1 & - & - \\
"USB" & - & - & 160 & - & - & - \\
"LSB" & - & - & - & 159 & 1 & - \\
"FM" & - & - & - & - & 160 & - \\
"PM" & - & 1 & 1 & 1 & - & 157 \\
Total & 160 & 160 & 161 & 161 & 161 & 157 \\
\hline
\end{tabular}

Table 5. Performance of the proposed method for FEM-1

\begin{tabular}{ccccccc}
\hline \multirow{2}{*}{ Actual } & \multicolumn{5}{c}{ Estimated } \\
\cline { 2 - 7 } & "AM" & "DSB" & "USB" & "LSB" & "FM" & "PM" \\
\hline "AM" & 155 & 2 & 1 & - & 2 & - \\
"DSB" & 2 & 150 & 2 & 4 & 1 & 1 \\
"USB" & - & 3 & 154 & 1 & 2 & - \\
"LSB" & 1 & 2 & - & 155 & 1 & 1 \\
"FM" & - & - & 3 & 2 & 155 & - \\
"PM" & - & 2 & - & 1 & - & 157 \\
Total & 158 & 159 & 160 & 163 & 161 & 159 \\
\hline
\end{tabular}

Table 6. Performance of the proposed method for FEM-3

\begin{tabular}{ccccccc}
\hline \multirow{2}{*}{ Actual } & \multicolumn{7}{c}{ Estimated } \\
\cline { 2 - 7 } & "AM" & "DSB" & "USB" & "LSB" & "FM" & "PM" \\
\hline "AM" & 152 & 3 & 2 & 2 & - & 1 \\
"DSB" & - & 153 & 2 & 2 & 1 & 2 \\
"USB" & 2 & - & 157 & - & - & 1 \\
"LSB" & 1 & 2 & - & 155 & 1 & 1 \\
"FM" & 2 & 2 & 1 & 1 & 153 & 1 \\
"PM" & 1 & - & - & - & 1 & 158 \\
Total & 158 & 160 & 161 & 160 & 156 & 164 \\
\hline
\end{tabular}


Table 7. Performance of the proposed method for FEM-4

\begin{tabular}{ccccccc}
\hline \multirow{2}{*}{ Actual } & \multicolumn{7}{c}{ Estimated } \\
\cline { 2 - 7 } & "AM" & "DSB" & "USB" & "LSB" & "FM" & "PM" \\
\hline "AM" & 155 & 2 & 1 & - & - & 1 \\
"DSB" & - & 157 & 1 & 2 & - & - \\
"USB" & 3 & 1 & 149 & 1 & 3 & 3 \\
"LSB" & 1 & 2 & - & 152 & 3 & 2 \\
"FM" & - & - & 3 & 1 & 154 & 2 \\
"PM" & - & 1 & - & - & 3 & 156 \\
Total & 159 & 163 & 154 & 156 & 163 & 164 \\
\hline
\end{tabular}

Table 8. Performance of the proposed method for FEM-5

\begin{tabular}{ccccccc}
\hline \multirow{2}{*}{ Actual } & \multicolumn{7}{c}{ Estimated } \\
\cline { 2 - 7 } & "AM" & "DSB" & "USB" & "LSB" & "FM" & "PM" \\
\hline "AM" & 154 & 1 & - & 2 & 1 & 2 \\
"DSB" & - & 155 & - & 1 & 2 & 2 \\
"USB" & 2 & 2 & 152 & 3 & 1 & - \\
"LSB" & - & 3 & 2 & 152 & 1 & 2 \\
"FM" & 1 & 1 & - & 2 & 156 & - \\
"PM" & 5 & 3 & - & 2 & 2 & 148 \\
Total & 162 & 165 & 154 & 162 & 163 & 154 \\
\hline
\end{tabular}

Table 9. Performance of the proposed method for FEM-6

\begin{tabular}{ccccccc}
\hline \multirow{2}{*}{ Actual } & \multicolumn{7}{c}{ Estimated } \\
\cline { 2 - 7 } & "AM" & "DSB" & "USB" & "LSB" & "FM" & "PM" \\
\hline "AM" & 152 & 3 & - & 1 & 2 & 2 \\
"DSB" & 1 & 154 & - & 1 & 1 & 3 \\
"USB" & 1 & 3 & 150 & 2 & 2 & 2 \\
"LSB" & - & 2 & 2 & 153 & 1 & 2 \\
"FM" & 2 & 1 & 1 & 2 & 154 & - \\
"PM" & 2 & 2 & 1 & 1 & - & 154 \\
Total & 158 & 165 & 154 & 160 & 160 & 163 \\
\hline
\end{tabular}

Table 10. Performance of the proposed method for FEM-7

\begin{tabular}{ccccccc}
\hline \multirow{2}{*}{ Actual } & \multicolumn{7}{c}{ Estimated } \\
\cline { 2 - 7 } & "AM" & "DSB" & "USB" & "LSB" & "FM" & "PM" \\
\hline "AM" & 153 & 3 & 2 & - & - & 2 \\
"DSB" & 1 & 155 & 1 & 2 & - & 1 \\
"USB" & 2 & 1 & 152 & 1 & 2 & 2 \\
"LSB" & 1 & 2 & - & 154 & 2 & 1 \\
"FM" & 4 & - & 2 & 1 & 151 & 2 \\
"PM" & 1 & 1 & - & - & 2 & 156 \\
Total & 162 & 162 & 157 & 158 & 157 & 164 \\
\hline
\end{tabular}

Table 11. Performance of the proposed method for FEM-8

\begin{tabular}{ccccccc}
\hline \multirow{2}{*}{ Actual } & \multicolumn{7}{c}{ Estimated } \\
\cline { 2 - 7 } & "AM" & "DSB" & "USB" & "LSB" & "FM" & "PM" \\
\hline "AM" & 152 & 3 & 2 & - & 1 & 2 \\
"DSB" & 1 & 153 & 2 & 2 & 1 & 1 \\
"USB" & 1 & 1 & 155 & 1 & 1 & 1 \\
"LSB" & 1 & 1 & - & 156 & 1 & 1 \\
"FM" & - & - & - & 1 & 157 & 2 \\
"PM" & 1 & 1 & 1 & 1 & 1 & 155 \\
Total & 156 & 159 & 160 & 161 & 162 & 162 \\
\hline
\end{tabular}


Table 12. Performance of the proposed method for FEM-9

\begin{tabular}{ccccccc}
\hline \multirow{2}{*}{ Actual } & \multicolumn{7}{c}{ Estimated } \\
\cline { 2 - 7 } & "AM" & "DSB" & "USB" & "LSB" & "FM" & "PM" \\
\hline "AM" & 155 & 1 & 1 & 1 & 1 & 1 \\
"DSB" & 1 & 154 & - & 2 & 2 & 1 \\
"USB" & - & - & 157 & 1 & - & 2 \\
"LSB" & 1 & 1 & - & 156 & 1 & 1 \\
"FM" & - & - & - & 1 & 157 & 2 \\
"PM" & 1 & 1 & 1 & 1 & 1 & 155 \\
Total & 158 & 157 & 159 & 162 & 162 & 162 \\
\hline
\end{tabular}

Table 13. Performance of the proposed method for FEM-10

\begin{tabular}{ccccccc}
\hline \multirow{2}{*}{ Actual } & \multicolumn{7}{c}{ Estimated } \\
\cline { 2 - 7 } & "AM" & "DSB" & "USB" & "LSB" & "FM" & "PM" \\
\hline "AM" & 152 & 3 & - & 1 & 2 & 2 \\
"DSB" & - & 157 & 1 & 2 & - & - \\
"USB" & - & 3 & 154 & 1 & 2 & - \\
"LSB" & - & - & - & 159 & 1 & - \\
"FM" & - & - & 3 & 2 & 155 & - \\
"PM" & 1 & 1 & 1 & 1 & 1 & 155 \\
Total & 153 & 163 & 159 & 166 & 161 & 157 \\
\hline
\end{tabular}

Table 14. Performance of the proposed method for FEM-11

\begin{tabular}{ccccccc}
\hline \multirow{2}{*}{ Actual } & \multicolumn{7}{c}{ Estimated } \\
\cline { 2 - 7 } & "AM" & "DSB" & "USB" & "LSB" & "FM" & "PM" \\
\hline "AM" & 152 & 3 & 2 & - & 1 & 2 \\
"DSB" & 1 & 154 & - & 2 & 2 & 1 \\
"USB" & 2 & 1 & 152 & 1 & 2 & 2 \\
"LSB" & - & 3 & 2 & 152 & 1 & 2 \\
"FM" & - & - & 3 & 2 & 155 & - \\
"PM" & 1 & 1 & - & - & 2 & 156 \\
Total & 155 & 162 & 159 & 157 & 163 & 163 \\
\hline
\end{tabular}

Table 15. Performance of the proposed method for FEM-12

\begin{tabular}{ccccccc}
\hline \multirow{2}{*}{ Actual } & \multicolumn{7}{c}{ Estimated } \\
\cline { 2 - 7 } & "AM" & "DSB" & "USB" & "LSB" & "FM" & "PM" \\
\hline "AM" & 155 & 1 & 1 & 1 & 1 & 1 \\
"DSB" & 1 & 155 & 1 & 2 & - & 1 \\
"USB" & 2 & 1 & 152 & 1 & 2 & 2 \\
"LSB" & - & 2 & 2 & 153 & 1 & 2 \\
"FM" & 1 & 1 & - & 2 & 156 & - \\
"PM" & - & 1 & - & - & 3 & 156 \\
Total & 159 & 161 & 156 & 159 & 163 & 162 \\
\hline
\end{tabular}

Table 16. Performance of the proposed method for FEM-13

\begin{tabular}{ccccccc}
\hline \multirow{2}{*}{ Actual } & \multicolumn{6}{c}{ Estimated } \\
\cline { 2 - 7 } & "AM" & "DSB" & "USB" & "LSB" & "FM" & "PM" \\
\hline "AM" & 152 & 3 & - & 1 & 2 & 2 \\
"DSB" & 1 & 153 & 2 & 2 & 1 & 1 \\
"USB" & 2 & 2 & 152 & 3 & 1 & - \\
"LSB" & - & 2 & 2 & 153 & 1 & 2 \\
"FM" & 1 & 1 & - & - & 154 & 4 \\
"PM" & 1 & 3 & 1 & 2 & 1 & 152 \\
Total & 157 & 164 & 157 & 161 & 160 & 161 \\
\hline
\end{tabular}


Table 17. Performance of the proposed method for FEM-14

\begin{tabular}{ccccccc}
\hline \multirow{2}{*}{ Actual } & \multicolumn{6}{c}{ Estimated } \\
\cline { 2 - 7 } & "AM" & "DSB" & "USB" & "LSB" & "FM" & "PM" \\
\hline "AM" & 152 & 3 & - & 1 & 2 & 2 \\
"DSB" & 1 & 155 & 1 & 2 & - & 1 \\
"USB" & 2 & 1 & 152 & 1 & 2 & 2 \\
"LSB" & - & 2 & 2 & 153 & 1 & 2 \\
"FM" & 4 & - & 2 & 1 & 151 & 2 \\
"PM" & 1 & - & - & - & 1 & 158 \\
Total & 161 & 161 & 157 & 158 & 157 & 167 \\
\hline
\end{tabular}

Table 18. Performance of the proposed method for FEM-15

\begin{tabular}{ccccccc}
\hline \multirow{2}{*}{ Actual } & \multicolumn{7}{c}{ Estimated } \\
\cline { 2 - 7 } & "AM" & "DSB" & "USB" & "LSB" & "FM" & "PM" \\
\hline "AM" & 151 & 2 & 2 & 3 & 1 & 1 \\
"DSB" & 1 & 155 & 1 & 2 & - & 1 \\
"USB" & - & - & 157 & 1 & - & 2 \\
"LSB" & 2 & 2 & 1 & 149 & 3 & 3 \\
"FM" & 3 & 1 & 1 & 2 & 152 & 1 \\
"PM" & 2 & 3 & 1 & - & 1 & 153 \\
Total & 159 & 163 & 163 & 157 & 157 & 161 \\
\hline
\end{tabular}

Table 19. Performance of the proposed method for FEM-16

\begin{tabular}{ccccccc}
\hline \multirow{2}{*}{ Actual } & \multicolumn{6}{c}{ Estimated } \\
\cline { 2 - 7 } & "AM" & "DSB" & "USB" & "LSB" & "FM" & "PM" \\
\hline "AM" & 154 & 2 & - & 1 & 1 & 2 \\
"DSB" & 1 & 153 & 2 & 2 & 1 & 1 \\
"USB" & 2 & 1 & 152 & 1 & 2 & 2 \\
"LSB" & 1 & 1 & - & 156 & 1 & 1 \\
"FM" & 2 & 2 & 1 & 1 & 153 & 1 \\
"PM" & 1 & 1 & 1 & 1 & 1 & 155 \\
Total & 161 & 160 & 156 & 162 & 159 & 162 \\
\hline
\end{tabular}

Table 20. Performance of the proposed method for FEM-17

\begin{tabular}{ccccccc}
\hline \multirow{2}{*}{ Actual } & \multicolumn{7}{c}{ Estimated } \\
\cline { 2 - 7 } & "AM" & "DSB" & "USB" & "LSB" & "FM" & "PM" \\
\hline "AM" & 151 & 3 & 4 & 2 & - & - \\
"DSB" & 2 & 154 & 3 & - & - & 1 \\
"USB" & 2 & 1 & 149 & 3 & 2 & 3 \\
"LSB" & 1 & 2 & 1 & 155 & 1 & - \\
"FM" & 3 & 1 & - & 1 & 154 & 1 \\
"PM" & - & 3 & 1 & - & 1 & 155 \\
Total & 159 & 164 & 158 & 161 & 158 & 160 \\
\hline
\end{tabular}

Table 21. Performance of the proposed method for FEM-18

\begin{tabular}{ccccccc}
\hline \multirow{2}{*}{ Actual } & \multicolumn{7}{c}{ Estimated } \\
\cline { 2 - 7 } & "AM" & "DSB" & "USB" & "LSB" & "FM" & "PM" \\
\hline "AM" & 155 & 1 & 1 & 2 & - & 1 \\
"DSB" & 1 & 156 & 1 & 1 & 1 & - \\
"USB" & 2 & 1 & 151 & 1 & 3 & 2 \\
"LSB" & 1 & - & 3 & 153 & 1 & 2 \\
"FM" & 3 & 1 & - & - & 149 & 7 \\
"PM" & 5 & 1 & 1 & 2 & 1 & 150 \\
Total & 167 & 160 & 157 & 159 & 155 & 162 \\
\hline
\end{tabular}


Table 22. Performance of the proposed method for FEM-19

\begin{tabular}{ccccccc}
\hline \multirow{2}{*}{ Actual } & \multicolumn{7}{c}{ Estimated } \\
\cline { 2 - 7 } & "AM" & "DSB" & "USB" & "LSB" & "FM" & "PM" \\
\hline "AM" & 153 & 3 & 2 & 2 & - & - \\
"DSB" & 1 & 153 & 3 & 2 & - & 1 \\
"USB" & - & 1 & 155 & - & 2 & 2 \\
"LSB" & 1 & 2 & 2 & 152 & 2 & 1 \\
"FM" & - & 2 & 3 & 1 & 154 & - \\
"PM" & 2 & 2 & 1 & - & - & 155 \\
Total & 157 & 162 & 167 & 158 & 156 & 158 \\
\hline
\end{tabular}

Table 23. Performance of the proposed method for FEM-20

\begin{tabular}{ccccccc}
\hline \multirow{2}{*}{ Actual } & \multicolumn{7}{c}{ Estimated } \\
\cline { 2 - 7 } & "AM" & "DSB" & "USB" & "LSB" & "FM" & "PM" \\
\hline "AM" & 155 & 3 & 1 & 1 & - & - \\
"DSB" & 1 & 153 & 3 & 2 & - & 1 \\
"USB" & - & 1 & 157 & - & 1 & 1 \\
"LSB" & 1 & 2 & 2 & 154 & 1 & - \\
"FM" & - & 2 & 3 & 1 & 153 & 1 \\
"PM" & 2 & 1 & 1 & - & 1 & 155 \\
Total & 159 & 162 & 167 & 158 & 156 & 158 \\
\hline
\end{tabular}

From above tables, it is shown that FEM-2 has the best classification accuracy for analog modulation types. Table 24 shows the classification accuracy for each FEM.

Table 24. Performance comparison between the proposed method and Ref. [11] for analog modulated signal

\begin{tabular}{ccc}
\multicolumn{3}{c}{ classification } \\
\hline & \multicolumn{2}{c}{ Classification accuracy (\%) } \\
\cline { 2 - 3 } FEMs & This study & Ref. [11] \\
\hline FEM-1 & 96.45 & 99.27 \\
FEM-2 & 99.27 & 98.22 \\
FEM-3 & 96.66 & 97.60 \\
FEM-4 & 96.14 & 95.52 \\
FEM-5 & 95.52 & 95.31 \\
FEM-6 & 95.52 & 95.31 \\
FEM-7 & 95.93 & 97.91 \\
FEM-8 & 96.66 & 95.52 \\
FEM-9 & 97.29 & 93.33 \\
FEM-10 & 97.08 & 96.35 \\
FEM-11 & 95.93 & 96.04 \\
FEM-12 & 96.56 & 93.33 \\
FEM-13 & 95.41 & 93.95 \\
FEM-14 & 95.93 & 97.91 \\
FEM-15 & 95.52 & 94.06 \\
FEM-16 & 96.14 & 97.50 \\
FEM-17 & 95.62 & 95.72 \\
FEM-18 & 95.20 & 96.66 \\
FEM-19 & 96.04 & 92.08 \\
FEM-20 & 96.56 & 94.68 \\
Mean accuracy (\%) & 96.2715 & 95.81 \\
\hline
\end{tabular}

The results obtained from this table show that the performance of the proposed method is 96.2715. Traditional approaches such as "Decision Theory", "Statistical Pattern Recognition"; It is disadvantageous due to large-size feature vectors, complex algorithms and the need for large storage space [31]. The proposed method eliminates drawbacks mentioned above. Therefore, the proposed method is an alternative approach to conventional approaches such as "Decision Theory", "Statistical Pattern Recognition", presented for classification of AMS. However, a poor classification performance can be obtained for signals with higher SNR when ELM classifier is trained by using signals with lower 
SNR [32]. Both smaller size and more efficiently feature vector and a compact set of features are obtained by using DWT. Thus, the classification performance is improved by using DWT. Performance of the proposed method for analog modulated signal classification is also presented in Table 24 as compared with Ref [11]. This table reveals that the proposed method shows a more effective classification performance than results of Ref [11].

\section{Conclusion}

This study proposes a method based on DWT-ELM for analog modulation classification. For this aim, a modulated "real voice signal" using the 6 different "AMS" schemes as "AM", "DSB", "USB", "LSB", "FM", and "PM" is used for experimental study. The classification performance of the proposed method is evaluated for 20 different feature extraction algorithms. Further, "White Gaussian Noise" is added to analog signals. Same experiments are conducted for analog signals added white Gaussian noise. Results from this study were compared with the previous study. However, from the experimental results, it can be said that the proposed method has a better performance against noise.

\section{Author's Contributions}

All contributions belong to the author in this paper.

\section{Statement of Conflicts of Interest}

No potential conflict of interest was reported by the authors.

\section{Statement of Research and Publication Ethics}

The authors declare that this study complies with Research and Publication Ethics.

\section{References}

[1] Fabrizi P.M., Lopes L.B., Lockhart G.B. 1986. Receiver recognition of analogue modulation types. In: IERE conference on radio receiver and associated systems, 1-4 July, University of Bangor, Wales, 135-140.

[2] Chan Y., Gadbois L., Yansouni P. 1985. Identification of the modulation type of a signal. In: ICASSP '85. IEEE International Conference on Acoustics, Speech, and Signal Processing, 26-29 April, Tampa, FL, USA, 10: 838-841.

[3] Nagy P.A.J. 1994. Analysis of a method for classification of analogue modulated radio signals. in In: European association for signal processing VII conference, Edinburgh, Scotland, 10151018.

[4] Jovanic S.D., Doroslovacki M.I., Dragosevic M.V. 1994. Recognition of low modulation index AM signals in additive Gaussian noise, in Recognition of low modulation index AM signals in additive Gaussian noise. In European Association for Signal Processing V Conference, Edinburgh, Scotland, 1923-1926.

[5] Al-Jalili Y.O. 1995. Identification algorithm of upper sideband and lower sideband SSB signals. Signal Processing, 42 (2): 207-213.

[6] Azzouz E.E., Nandi A.K. 1996. Procedure for automatic recognition of analogue and digital modulations. IEE Proc.-Commun., 143 (5): 259.

[7] Wong M.L.D., Nandi A.K. 2004. Automatic digital modulation recognition using artificial neural network and genetic algorithm. Signal Processing, 84 (2): 351-365.

[8] Kavalov D., Kalinin V. 2001. Improved noise characteristics of a SAW artificial neural network RF signal processor for modulation recognition. In: IEEE Ultrasonics Symposium. Proceedings. An International Symposium (Cat. No.01CH37263), 7-10 October, Atlanta, GA, USA, 1: 19-22.

[9] Kremer S.C., Shiels J. 1997. A testbed for automatic modulation recognition using artificial neural networks. In: CCECE '97, Canadian Conference on Electrical and Computer Engineering. Engineering Innovation: Voyage of Discovery. Conference Proceedings, 1: 67-70. 
[10] Nandi A.K., Azzouz E.E. 1998. Algorithms for automatic modulation recognition of communication signals. IEEE Trans. Commun., 46 (4): 431-436.

[11] Avci E. 2007. Performance comparison of wavelet families for analog modulation classification using expert discrete wavelet neural network system. Expert Syst. Appl., 33 (1): 23-35.

[12] Zhang J., Walter G.G., Miao Y., Wayne L.W.N. 1995. Wavelet neural networks for function learning. IEEE Trans. Signal Process., 43 (6): 1485-1497.

[13] Kociołek M., Materka A., Strzelecki M., Szczypiński P. 2001. Discrete Wavelet Transform Derived Features for Digital Image Texture Analysis. Proc. of Interational Conference on Signals and Electronic Systems, 18-21 September, Lodz, Poland, 18-21.

[14] Avci E., Turkoglu I., Poyraz M. 2005. Intelligent target recognition based on wavelet packet neural network. Expert Syst. Appl., 29 (1): 175-182.

[15] Thuillard M. 2000. A Review of Wavelet Networks, Wavenets, Fuzzy Wavenets and their Applications. Advances in Computational Intelligence and Learning, 43-60.

[16] Wesfreid E., Wickerhauser M.V. 1993. Adapted local trigonometric transforms and speech processing. IEEE Trans. Signal Process., 41 (12): 3596-3600.

[17] Huang G.B., Wang D. H., Lan Y. 2011. Extreme learning machines: a survey. Int. J. Mach. Learn. Cybern., 2 (2): 107-122.

[18] Huang G.B., Zhou H., Ding X., Zhang R. 2012. Extreme Learning Machine for Regression and Multiclass Classification. IEEE Trans. Syst. Man, Cybern. Part B, 42 (2): 513-529.

[19] Bengio Y. 2009. Learning Deep Architectures for AI. Found. Trends® Mach. Learn., 2 (1): 1127.

[20] Daliri M.R. 2012. A hybrid automatic system for the diagnosis of lung cancer based on genetic algorithm and fuzzy extreme learning machines. J. Med. Syst., 36 (2): 1001-1005.

[21] Qu Y., Shang C., Wu W., Shen Q. 2011. Evolutionary fuzzy extreme learning machine for mammographic risk analysis. Int. J. Fuzzy Syst., 13 (4): 282-291.

[22] Xu Y., Dong Z.Y., Meng K., Wong K.P., Zhang R. 2013. Short-term load forecasting of Australian National Electricity Market by an ensemble model of extreme learning machine. IET Gener. Transm. Distrib., 7 (4): 391-397.

[23] Avci E., Coteli R. 2012. A new automatic target recognition system based on wavelet extreme learning machine. Expert Syst. Appl., 39 (16): 12340-12348.

[24] Cao J., Lin Z., Huang G. 2010. Composite function wavelet neural networks with extreme learning machine. Neurocomputing, 73 (7): 1405-1416.

[25] Malathi V., Marimuthu N.S., Baskar S. 2010. Intelligent approaches using support vector machine and extreme learning machine for transmission line protection. Neurocomputing, 73 (10): 21602167.

[26] Malathi V., Marimuthu N.S., Baskar S., Ramar K. 2011. Application of extreme learning machine for series compensated transmission line protection. Eng. Appl. Artif. Intell., 24 (5): 880-887.

[27] Banerjee K.S. 1973. Generalized Inverse of Matrices and Its Applications. Technometrics, 15 (1): 197-197.

[28] Schmidt W.F., Kraaijveld M.A., Duin R.P.W. 1992. Feed Forward Neural Networks with Random Weights. In International Conference on Pattern Recognition, August, 1-4.

[29] White H. 1989. An additional hidden unit test for neglected nonlinearity in multilayer feedforward networks. International Joint Conference on Neural Networks, 2: 451-455.

[30] White H. 2006. Approximate Nonlinear Forecasting Methods. Handbook of Economic Forecasting, 459-512.

[31] Azzouz E.E., Nandi A.K. 1997. Automatic modulation recognition-II. J. Franklin Inst., 334 (2): 275-305.

[32] Wu Z., Ren G., Wang X., Zhao Y. 2004. Automatic Digital Modulation Recognition Using Wavelet Transform and Neural Networks. Springer, Berlin, Heidelberg, 936-940. 